KEK-TH-1171

August 2007

\title{
Higgs Particle: The Origin of Mass
}

\author{
Yasuhiro Okada ${ }^{(a, b)} 1$ \\ (a) Theory Group, KEK, Oho 1-1 Tsukuba, 305-0801, Japan \\ (b) The Graduate University for Advanced Studies (Sokendai), \\ Oho 1-1 Tsukuba, 305-0801, Japan
}

\begin{abstract}
The Higgs particle is a new elementary particle predicted in the Standard Model of the elementary particle physics. It plays a special role in the theory of mass generation of quarks, leptons, and gauge bosons. In this article, theoretical issues on the Higgs mechanism are first discussed, and then experimental prospects on the Higgs particle study at the future collider experiments, LHC and ILC, are reviewed. The Higgs coupling determination is an essential step to establish the mass generation mechanism, which could lead to a deeper understanding of particle physics.
\end{abstract}

\footnotetext{
${ }^{1}$ E-mail: yasuhiro.okada@kek.jp
} 


\section{Introduction}

Current understanding of the elementary particle physics is based on two important concepts, gauge invariance and spontaneous symmetry breaking. Out of four fundamental interactions of Nature, namely strong, weak and electromagnetic and gravity interactions, three of them except for gravity are described on the same footing in terms of gauge theory. The gauge group corresponding to the strong interaction is $S U(3)$, and the weak and the electromagnetic interactions arise from $S U(2)$ and $U(1)$ groups and are called the electroweak interaction. Once quarks and leptons are assigned in proper representations of the three gauge groups, all properties of the three fundamental interactions are determined from the requirement of gauge invariance.

For more than thirty years, high energy experiments have been testing various aspects of gauge symmetry and have established gauge invariance as a fundamental principle of Nature. We have discovered gauge bosons mediating the three interactions, namely gluon for the strong interaction, $\mathrm{W}$ and $\mathrm{Z}$ bosons for the electroweak interaction. The couplings between quarks/leptons and gauge bosons have been precisely measured at the CERN LEP and SLAC SLC experiments, and we have confirmed the assignments of the gauge representations for quarks and leptons.

The gauge principle alone, however, cannot describe the known structure of the elementary particle physics. In the Standard Model of the elementary particle physics, all quarks, leptons and gauge bosons are first introduced as massless fields. In order to generate masses for these particles, the $S U(2) \times U(1)$ symmetries have to be broken spontaneously.

Spontaneous symmetry breaking itself is not new for particle physics [1, 2]. The theory of the strong interaction, QCD, possesses an approximate symmetry among three light quarks called chiral symmetry. The vacuum of QCD corresponds to a state where quark and anti-quark pair is condensed, and the chiral symmetry is broken spontaneously. As a consequence, pseudo scalar mesons such as pions and kaons are light compared to the typical energy scale of the strong interaction since they behave approximately as Nambu-Goldstone bosons, a characteristic signature of spontaneous symmetry breaking.

In the case of the electroweak symmetry, it is shown theoretically that the NambuGoldstone bosons associated with spontaneous breakdown are absorbed by gauge bosons, providing the mass generation mechanism for gauge bosons (Higgs mech- 
anism) [3]. Although we are now quite sure that this is the mechanism for gauge boson mass generation, we know little about how the symmetry breaking occurs, or what is dynamics behind the Higgs mechanism. Clearly, we need a new interaction other than four known fundamental forces, but we do not know what it is. The goal of the Higgs physics is to answer this question.

In this article, I would like to explain what are theoretical issues of the Higgs sector, what is expected at the future collider experiments, LHC and ILC, and what would be impacts of the Higgs physics on a deeper understanding of the particle physics.

\section{Higgs boson in the Standard Model}

In the Standard Model, a single Higgs doublet field is included for the symmetry breaking of the $S U(2) \times U(1)$ gauge groups. This was introduced in S. Weinberg's 1967 paper "A Model of Leptons" [4], and is the simplest possibility for generating the gauge boson masses.

The Higgs potential is given by

$$
V(\Phi)=-\mu^{2}|\Phi|^{2}+\lambda|\Phi|^{4}
$$

where the two component complex field is defined as

$$
\Phi(x)=\left(\begin{array}{c}
\phi(x)^{+} \\
\phi(x)^{0}
\end{array}\right) .
$$

In order for the stability of the vacuum the parameter $\lambda$ must be positive. The coefficient of the quadratic term, on the other hand, can be either sign. In fact, if the sign is negative, namely $\mu^{2}>0$, the origin of the potential is unstable, and the vacuum state corresponds to a non-zero value of the $\Phi$ field. The states satisfying $\left|\phi^{+}\right|^{2}+\left|\phi^{0}\right|^{2}=\frac{\mu^{2}}{2 \lambda} \equiv \frac{v^{2}}{2}$ are degenerate minimum of the potential. We can choose the vacuum expectation value in the $\phi^{0}$ direction, $\left\langle\phi^{0}\right\rangle=\frac{v}{\sqrt{2}}$, and then there are three massless modes corresponding to the flat directions of the potential (NambuGoldstone modes). When the symmetry is a gauge symmetry, these massless particles disappear from the physical spectrum, and become longitudinal components of massive gauge bosons. This is seen most clearly if we take the "Unitary gauge" where the Nambu-Goldstone modes are removed by an appropriate gauge transformation. The kinetic term of the scalar field is defined as $\left|D_{\mu} \Phi\right|^{2}=\left|\left(\partial_{\mu}+g \frac{\tau^{a}}{2} W_{\mu}^{a}+\frac{g^{\prime}}{2} B_{\mu}\right) \Phi\right|^{2}$, 
and the gauge boson mass terms are obtained by substituting the vacuum expectation value into $\Phi(x)$.

Mass terms of quarks and leptons are also generated through interactions with the Higgs field. This follows from the chiral structure of quarks and leptons. Since the discovery of the parity violation in the weak interaction [5], chiral projected fermions (Weyl fermions) instead of Dirac fermions have been considered as building blocks of a particle physics model. In particular, only left-handed quarks and leptons are assigned as $S U(2)$ doublets because the weak interaction has a V(vector)-A(axial vector) current structure. Right-handed counter parts are singlet under the $S U(2)$ gauge group. This unbalance in the $S U(2)$ quantum number assignment forbids us to write direct mass terms for quarks and leptons: the only possible way to generate mass terms is to introduce Yukawa couplings with help of the $\Phi$ field such as $y_{d} \Phi^{\dagger} \bar{d}_{R} q_{L}$ where $q_{L}=\left(u_{L}, d_{L}\right)^{T}$. After replacing $\Phi(x)$ by its vacuum expectation value, this term generates a mass of $y_{d} v / \sqrt{2}$ for down-type quarks. Similar mechanism works for up-type quarks and charged leptons.

There is one important prediction of this model. Since we introduce a twocomponent complex field and three real degrees of freedom are absorbed by gauge bosons, one scalar particle appears in the physical spectrum, which is called the Higgs particle (三 Higgs boson). In the Unitary gauge, interactions related to the Higgs boson can be obtained by replacing $v$ with $v+H(x)$ in the Lagrangian where $H(x)$ represents the Higgs boson. The mass of the Higgs boson is given by $m_{h}=\sqrt{2 \lambda} v$, which means that the Higgs boson becomes heavier if the Higgs self-coupling gets larger. In fact, this is a general property of the particle mass generation mechanism due to the Higgs field: A stronger interaction leads to a heaver particle. The mass formula for the W, Z bosons, quarks, leptons and the Higgs boson at the lowest order approximation with respect to coupling perturbation (i.e. tree-revel) are summarized in table 1 .

Table 1: Mass formula for elementary particles. $g, g^{\prime}, y_{f}$, and $\lambda$ are the $S U(2)$ and the $U(1)$ gauge coupling constants, the Yukawa coupling constant for a fermion $f$, and the Higgs self-coupling constant.

\begin{tabular}{|c|c|c|c|}
\hline W boson & Z boson & quarks, leptons & Higgs boson \\
\hline$\frac{g}{2} v$ & $\frac{\sqrt{g^{2}+g^{\prime 2}}}{2} v$ & $y_{f} \frac{v}{\sqrt{2}}$ & $\sqrt{2 \lambda} v$ \\
\hline
\end{tabular}




\section{Naturalness and Physics beyond the Standard Model}

Although the Higgs potential in Eq.1 is very simple and sufficient to describe a realistic model of mass generation, we think that this is not the final form of the theory but rather an effective description of a more fundamental theory. It is therefore important to know what is limitation of this description of the Higgs sector.

In renormalizable quantum field theories, the form of Lagrangian is specified by requirement for renormalizability. In the case of the Higgs potential, quadratic and quartic terms are only renormalizable interactions. We can then consider two kinds of corrections to the potential. One is a calculable higher order correction within the Standard Model. For instance the correction from the top Yukawa coupling constant can be evaluated up to a desired accuracy applying renormalization procedure of field theory. Another type of corrections comes from outside of the present model, presumably from physics at some high energy scale. We cannot really compute these corrections until we know the more fundamental theory. In this sense, the present theory is considered as an effective theory below some cutoff energy scale $\Lambda$.

Although the effective theory cannot include all physical effects, it is still useful because unknown correction is expected to be suppressed by $(E / \Lambda)^{2}$ where $E$ is a typical energy scale under consideration. Therefore, as long as the cutoff scale is somewhat larger than $E$, the theory can make fairly accurate predictions. For example, the correction is $0\left(10^{-4}\right)$ when the cutoff scale is around $10 \mathrm{TeV}$ for physical processes in the $100 \mathrm{GeV}$ range. If the theory is valid up to the Planck scale $\left(\sim 10^{19}\right.$ $\mathrm{GeV}$ ) where the gravity interaction becomes as strong as the other gauge interactions, the correction becomes extremely small. In this way, an effective theory is useful description as long as we restrict ourself to the energy regime below the cutoff scale.

Once we take a point of view that the Higgs sector of the Standard Model is an effective description of a more complete theory below $\Lambda$, naturalness with regard to parameter fine-tuning becomes a serious problem. In particular, the quadratic divergence of the Higgs mass radiative correction is problematic, and this has been one of main motivations to introduce various models beyond the Standard Model.

In the Higgs potential in Eq,1 the only mass parameter is $\mu^{2}$. At the tree level, this parameter is related to the vacuum expectation value $v$ by $\mu^{2}=\lambda v^{2}$ where $v$ is known

to be about $246 \mathrm{GeV}$. $\left(v=\left(\sqrt{2} G_{F}\right)^{-\frac{1}{2}}\right.$, where $G_{F}$ is the Fermi constant representing the coupling constant of the weak interaction.) If we include the radiative correction, 
$\mu^{2}$ becomes a sum of two contributions $\mu_{0}^{2}+\delta \mu^{2}$ where $\mu_{0}^{2}$ is a bare mass term and $\delta \mu^{2}$ is the radiative correction. In the Standard Model, the top quark and gauge boson loop corrections are important and $\delta \mu^{2}$ from these sources are represented by a sum of terms of a form $C_{i} \frac{g_{i}^{2}}{(4 \pi)^{2}} \Lambda^{2}$ where $g_{i}$ is the top Yukawa coupling constant, or $U(1)$ or $S U(2)$ gauge coupling constant and $C_{i}$ are $O(1)$ coefficients. Since the radiative correction depends on the cutoff scale quadratically, the fine-tuning between the bare mass term and the radiative correction is necessary if the cutoff scale is much larger than $1 \mathrm{TeV}$. Roughly speaking, the fine-tuning at $1 \%$ level is necessary for $\Lambda=10$ $\mathrm{TeV}$. If the cutoff scale is close to the Planck scale, the degree of the fine-tuning is enormous: A tuning of one out of $10^{32}$ is required. This is the naturalness problem of the Standard Model, and sometime also called the hierarchy problem. This problem suggests that the description of the Higgs sector by the simple potential in Eq. 1] is not very satisfactory, and probably will be replaced by a more fundamental form at a higher energy scale.

Since the problem arises from the quadratic divergence in the renormalization of the Higgs mass terms, proposed solutions involve cancellation mechanism of such divergence. Supersymmetry [6] is a unique symmetry that guarantees complete cancellation of the quadratic divergence in scalar field mass terms. This is a new symmetry between bosons and fermions and the cancellation occurs between loop diagrams of bosons and fermions. Particle physics models based on supersymmetry such as supersymmetric grand unified theory (SUSY GUT) have been proposed and studied since early 1980's as a possible way out of the hierarchy problem [7]. In a realistic model of a supersymmetric extension of the Standard Model, we need to introduce new particles connected by supersymmetry to ordinary quarks, leptons, gauge bosons, and Higgs particles. In 1990's, precision studies on the Z boson were preformed at LEP and SLC experiments, and it was pointed out that three precisely measured coupling constants are consistent with the prediction of SUSY GUT, although the gauge coupling unification fails badly without supersymmetric partner particles [8]. Supersymmetry is also an essential ingredient of the superstring theory, a potential unified theory including gravity and gauge interactions. In this way, the supersymmetric model has become a promising candidate beyond the Standard Model. If supersymmetry realizes at or just above the $\mathrm{TeV}$ scale, it can provide a consistent and unified picture of the particle physics from the weak scale to the Planck scale.

An opposite idea for solution of the naturalness problem is considering that the cutoff scale is close to the electroweak scale. In particular, the Higgs field is consid- 
ered to be a composite state of more fundamental objects at a relatively low energy scale. The simplest form of this model is called the technicolor model [9] proposed in late 1970's, in which the cutoff scale is about $1 \mathrm{TeV}$. The technicolor model is however strongly constrained from precision tests of electroweak theory later at the LEP and SLC experiments [10], but there have been continuous attempts to construct a phenomenologically viable model of a composite Higgs field. Little Higgs models[11] are a recent proposal on this line, where the physical Higgs boson is dynamically formed by a new strong interaction around $10 \mathrm{TeV}$. An interesting feature of this model is that the quadratic divergence of the Higgs boson mass term is canceled by loop corrections due to new gauge bosons and a heavy partner of the top quark at one loop level. In this way the hierarchy problem between the electroweak scale and $10 \mathrm{TeV}$ is nicely solved.

In addition to supersymmetry and little Higgs models, there have been many proposals for TeV scale physics. Motivations for many of them are solving the naturalness problem of the Standard Model or explaining the large (apparent) hierarchy between the weak scale and the gravity scale. Examples are models with large extra dimensions [12], models with warped extra-dimensions [13], the Higgsless model[14], the twin Higgs model[15], and the inert Higgs model[16], the split-supersymmetry model[17], etc. All of these proposals involve some characteristic signals around a $\mathrm{TeV}$ region. These signals are important to choose a correct model at the $\mathrm{TeV}$ scale and clarify the mechanism of the electroweak symmetry breaking.

\section{Experimental Prospects of Higgs Physics}

Higgs physics is expected to be the center of the particle physics in coming years starting from the commissioning of the CERN LHC experiment. The first step will be a discovery of a new particle which is a candidate of the Higgs boson. We then study its properties in detail and compare them with the prediction of the Standard Model Higgs boson. We may be able to confirm that the discovered particle is the Higgs boson responsible for the mass generation for elementary particles. Another possibility would be to find some deviation from the Standard Model Higgs boson. Deviation could be something like small difference of production cross section and decay branching ratios from the Standard Model predictions, or more drastic new signals such as discovery of several Higgs states. At the same time, we may also find other new particles predicted in extensions of the Standard Model, for 
example supersymmetric particles in the supersymmetric model or the heavy gauge bosons and the top partner in the little Higgs model. In order to accomplish these goals we probably need several steps in collider experiments including LHC and ILC experiments and possible upgrades for these facilities.

If we restrict ourselves to the Higgs boson in the Standard Model, all physical properties are determined by one parameter, the Higgs boson mass. Present experimental lower bound for the mass of the Standard Model Higgs boson is $114.4 \mathrm{GeV}$ at the $95 \%$ confidence level, set by the direct Higgs boson search at LEP [18]. It is remarkable that we can also draw an upper bound from a global fit of electroweak precision data. Although a heavy Higgs boson means a large self-coupling $\lambda$, we have not seen any evidence of such a large coupling in physical observables related to $Z$ and $W$ gauge boson processes. The upper limit of the Standard Model Higgs boson is $166 \mathrm{GeV}$ at the $95 \%$ confidence level [19]. This implies that a relatively light Higgs boson is favored. If the Higgs boson turns out to be heaver than 200 $\mathrm{GeV}$, we would expect some additional new particles that have significant couplings to gauge bosons.

The decay branching ratios of the Higgs boson depends strongly on the Higgs boson mass, and therefore the discovery strategy for the Higgs boson at LHC differs for light and heavy Higgs bosons. The branching ratios for the Standard Model Higgs boson is shown in figure 11. Since the Higgs boson couples more strongly to a heaver particle, it tends to decay to heaver particles as long as kinematically allowed. For instance, the Higgs boson mostly decays into two gauge bosons if the Higgs boson mass is larger than $200 \mathrm{GeV}$, whereas the bottom and anti-bottom pair is the main decay mode for its mass less than $140 \mathrm{GeV}$. For this mass range, the Higgs boson search at LHC relies on other decay modes such as the loop-induced two photon decay mode, because two bottom modes are hidden by overwhelming QCD background processes. Detail simulation studies on the Higgs discovery at LHC have been performed, and it is shown that the Higgs boson can be found at LHC experiments within a few years for the entire mass region as long as the production and decay properties are similar to the Standard Model Higgs boson[20, 21]. Furthermore, information on the Higgs couplings is obtained with a higher luminosity. Estimated precision for coupling ratios are typically $0(10) \%$ [22].

ILC is a future electron-positron linear collider project proposed in the international framework [23]. One aspect of this facility is a Higgs factory. For instance, the number of produced Higgs bosons can be $0\left(10^{5}\right)$ in the first stage of experiments 


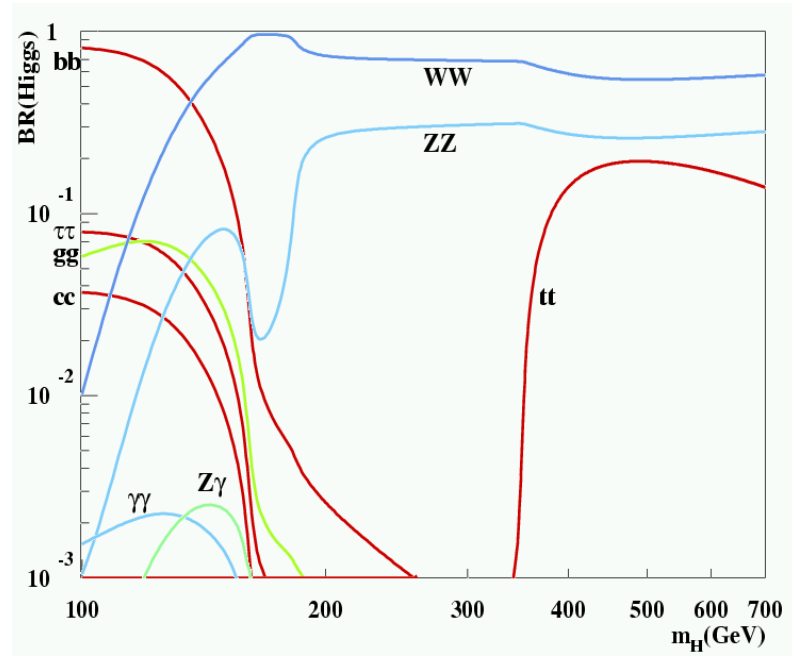

Figure 1: Decay branching ratio of the Standard Model Higgs boson as a function of its mass. $c, b, t, \tau$ represent charm, bottom, top quarks and tau lepton. $\gamma, g, W$, $Z$ are photon, gluon, $W$ and $Z$ bosons.

with the center-of-mass collider energy of $500 \mathrm{GeV}$. Under clean environment of the $e^{+} e^{-}$collider, precise determinations on the mass, quantum numbers, and coupling constants of the Higgs boson are possible. Typical production and decay processes are shown figures 2. Precision of the coupling constant determination reaches a few $\%$ level for Higgs- $W W$, Higgs- $Z Z$, and Higgs- $b \bar{b}$ couplings for the case of a relatively light Higgs boson. We can also measure the Higgs self-coupling from the double Higgs boson production process and the top Yukawa coupling from the Higgs- $t \bar{t}$ production. Figure 3 shows precision of the Higgs coupling constant determination for various particles at ILC. The proportionality between coupling constants and particle masses is a characteristic feature of the one Higgs doublet model where the particle mass formulas involve only one vacuum expectation value. An important feature of ILC experiments is that absolute values of these coupling constants can be determined in a model-independent way. This is crucial in establishing the mass generation mechanism for elementary particles.

The precise determination of the Higgs coupling constants is also useful to explore physics beyond the Standard Model. In some case, the Higgs boson coupling is modified from the Standard Model.

- The Higgs sector of supersymmetric models is different from the Standard Model. In any realistic supersymmetric model, the Higgs sector contains at 


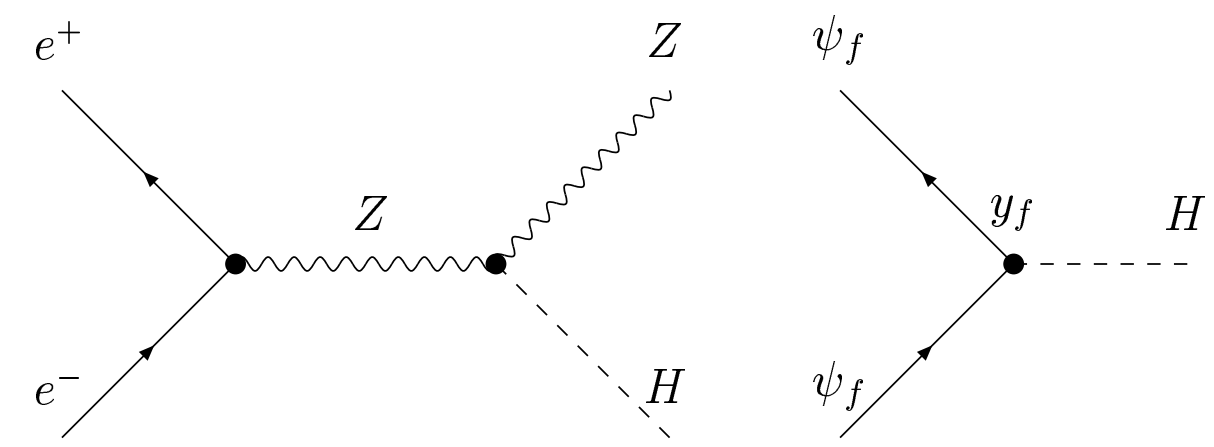

Figure 2: Production process of the Higgs boson at ILC (left) and Higgs boson decay to fermions (right).

lease two sets of doublet fields. In the minimal supersymmetric standard model (MSSM), in particular, the Higgs sector is a two Higgs doublet model. Furthermore, there is a rather strict theoretical upper bound for the the lightest neutral Higgs boson[25], which is about $130 \mathrm{GeV}$. Since this light boson plays a role of the usual Higgs particle, this particle may be the only Higgs particle discovered at LHC. In such case, the branching ratio measurement for the lightest neutral Higgs boson is useful to obtain information on the masses of heavy Higgs bosons [26, 27, 28]. In particular, the tau and bottom coupling constants show sizable enhancement if the heavy Higgs boson exists below $600 \mathrm{GeV}$. The ratio like $B(H \rightarrow W W) / B(H \rightarrow \tau \tau)$ is useful to determine the heavy Higgs mass scale indirectly.

- In models with extra dimensions, there appears a scalar field called Radion, corresponding to the size of the extra space dimension. Since Radion is a neutral scalar field, it can mix with the Higgs field. It is pointed out that Radion-Higgs mixing in the warped extra dimension model could reduce the magnitude of Yukawa coupling constants and $W W H$ and $Z Z H$ constant in a universal way [29]. In order to observe such effects, absolute coupling measurements at ILC are necessary.

- The two-gluon width of the Higgs boson is generated by loop diagrams, so that it can be a probe to virtual effects of new particles. The same is true for the two-photon width, the measurement of which is improved at the photon-photon collider option of ILC [30]. There are many new physics models where such loop effects are sizable. 
CouplingMass Relation

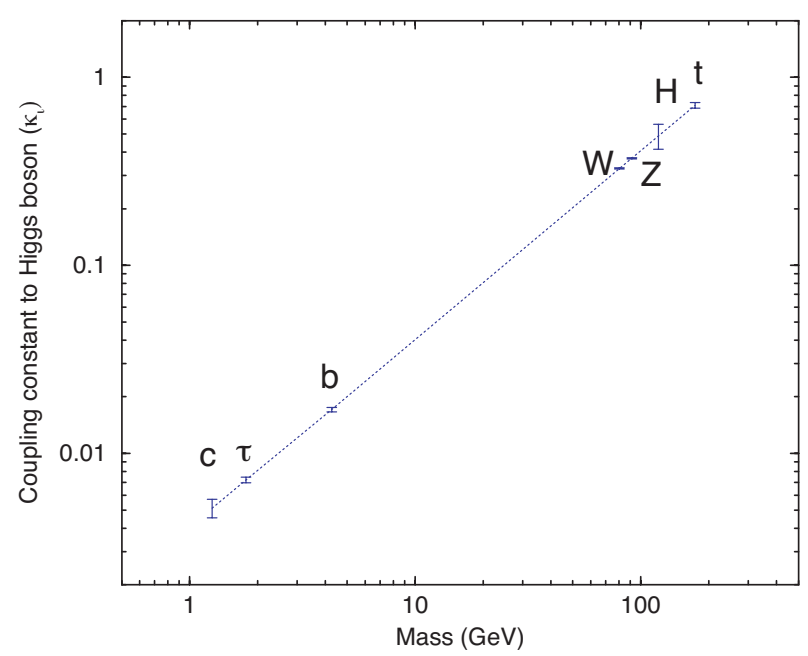

Figure 3: Precision of the coupling-constant determination for various particles at ILC with the integrated luminosity of $500 \mathrm{fb}^{-1}$. The Higgs boson mass is taken to be $120 \mathrm{GeV}$. For charm, tau, bottom, W, and Z coupling measurement, $\sqrt{s}=300 \mathrm{GeV}$ is assumed. $\sqrt{s}=500 \mathrm{GeV}(700 \mathrm{GeV})$ is taken for the triple Higgs $(t \bar{t} H)$ coupling measurement 24 .

- Explaining the baryon number of the Universe is one of most outstanding questions for particle physics in connection with cosmology [31]. One possibility is the electroweak baryogenesis scenario, in which the baryon number was generated at the electroweak phase transition. For a successful electroweak baryogenesis, the Higgs sector has to be extended from that of the minimal Standard Model to realize a strong first-order phase transition. The change of the Higgs potential can lead to observable effects in the triple Higgs coupling measurement [32, 33].

As we can see above examples, observations of new physics effects require precise determination of coupling constants. This will be an important goal of the future ILC experiment.

\section{Conclusions}

The Higgs sector is an unknown part of the particle physics model. Although a simple potential is assumed in the Standard Model, this description is supposed 
to be valid below some cutoff scale, beyond which the theory of the electroweak symmetry breaking takes in a more fundamental form. If the cutoff scale is as low as $1 \mathrm{TeV}$, some direct signals on new physics is likely to appear at LHC. If the cutoff scale is much larger, the fine-tuning of the Higgs boson mass term becomes a serious problem. Proposed solutions to this problem such as supersymmetry or little Higgs models also predict new physics signals at the $\mathrm{TeV}$ scale. These signals are targets of future collider experiments starting from LHC.

Experimental prospects for the Higgs physics are quite bright. The Higgs particle can be found and studied at LHC. At the proposed ILC, precise information on coupling constants between the Higgs boson and other particles will be obtained. These measurements are an essential step to establish the mass generation mechanism. At the same time, the precision measurement may reveal evidence of new force and/or new symmetry because these new physics is most probably related to the physics of electroweak symmetry breaking, i.e. the Higgs sector. In this way, the Higgs particle will play a special role in determining the future direction of the particle physics.

\section{Acknowledgment}

This work was supported in part by the Grant-in-Aid for Science Research, Ministry of Education, Culture, Sports, Science and Technology, Nos. 16081211 and 17540286.

\section{References}

[1] Y. Nambu and G. Jona-Lasinio, Phys. Rev. 122 (1961) 345.

[2] J. Goldstone, Nuovo Cim. 19 (1961) 154.

[3] P. W. Higgs, Phys. Lett. 12 (1964) 132; Phys. Rev. Lett. 13 (1964) 508; Phys. Rev. 145 (1966) 1156.

[4] S. Weinberg, Phys. Rev. Lett. 19 (1967) 1264.

[5] T. D. Lee and C. N. Yang, Phys. Rev. 104 (1956) 254.

[6] J. Wess and B. Zumino, Nucl. Phys. B 70 (1974) 39.

[7] N. Sakai, Z. Phys. C 11 (1981) 153; S. Dimopoulos and H. Georgi, Nucl. Phys. B 193 (1981) 150.

[8] P. Langacker and M. x. Luo, Phys. Rev. D 44 (1991) 817; U. Amaldi, W. de Boer and H. Furstenau, Phys. Lett. B 260 (1991) 447. 
[9] L. Susskind, Phys. Rev. D 20 (1979) 2619.

[10] M. E. Peskin and T. Takeuchi, Phys. Rev. Lett. 65 (1990) 964; M. Golden and L. Randall, Nucl. Phys. B 361 (1991) 3; B. Holdom and J. Terning, Phys. Lett. B 247 (1990) 88.

[11] N. Arkani-Hamed, A. G. Cohen, E. Katz, A. E. Nelson, T. Gregoire and J. G. Wacker, JHEP 0208 (2002) 021;. N. Arkani-Hamed, A. G. Cohen, E. Katz and A. E. Nelson, JHEP 0207 (2002) 034.

[12] N. Arkani-Hamed, S. Dimopoulos and G. R. Dvali, Phys. Lett. B 429 (1998) 263; I. Antoniadis, N. Arkani-Hamed, S. Dimopoulos and G. R. Dvali, Phys. Lett. B 436 (1998) 257; N. Arkani-Hamed, S. Dimopoulos and G. R. Dvali, Phys. Rev. D 59 (1999) 086004.

[13] L. Randall and R. Sundrum, Phys. Rev. Lett. 83 (1999) 3370.

[14] C. Csaki, C. Grojean, H. Murayama, L. Pilo and J. Terning, Phys. Rev. D 69 (2004) 055006; C. Csaki, C. Grojean, L. Pilo and J. Terning, Phys. Rev. Lett. 92 (2004) 101802.

[15] Z. Chacko, H. S. Goh and R. Harnik, Phys. Rev. Lett. 96 (2006) 231802.

[16] R. Barbieri, L. J. Hall and V. S. Rychkov, Phys. Rev. D 74 (2006) 015007.

[17] N. Arkani-Hamed and S. Dimopoulos, JHEP 0506 (2005) 073.

[18] R. Barate et al. [LEP Working Group for Higgs boson searches], Phys. Lett. B 565 (2003) 61.

[19] J. Alcaraz et al. The LEP Collaborations and the LEP electroweak working group, arXiv:hep-ex/0612034.

[20] ATLAS Collaboration, ATLAS Physics Technical Report, CERN-LHCC-99-14 and CERN-LHCC-99-15.

[21] CMS Collaboration, CMS Physics TDR, CERN/LHCC/2006-021.

[22] M. Duhrssen, S. Heinemeyer, H. Logan, D. Rainwater, G. Weiglein and D. Zeppenfeld, Phys. Rev. D 70 (2004) 113009.

[23] International Linear Collider home page, http://www.linearcollider.org/

[24] "GLC project: Linear collider for TeV physics," KEK-REPORT-2003-7.

[25] Y. Okada, M. Yamaguchi and T. Yanagida, Prog. Theor. Phys. 85 (1991) 1; J. R. Ellis, G. Ridolfi and F. Zwirner, Phys. Lett. B 257 (1991) 83; H. E. Haber and R. Hempfling, Phys. Rev. Lett. 66 (1991) 1815. 
[26] K. Abe et al. [ACFA Linear Collider Working Group], "Particle physics experiments at JLC," arXiv:hep-ph/0109166.

[27] J. A. Aguilar-Saavedra et al. [ECFA/DESY LC Physics Working Group], "TESLA Technical Design Report Part III: Physics at an e+e- Linear Collider," arXiv:hep-ph/0106315.

[28] G. Weiglein et al. [LHC/LC Study Group], "Physics interplay of the LHC and the ILC," Phys. Rept. 426 (2006) 47 arXiv:hep-ph/0410364.

[29] J. L. Hewett and T. G. Rizzo, JHEP 0308 (2003) 028; D. Dominici, B. Grzadkowski, J. F. Gunion and M. Toharia, Nucl. Phys. B 671 (2003) 243.

[30] B. Badelek et al. [ECFA/DESY Photon Collider Working Group], Int. J. Mod. Phys. A 19 (2004) 5097.

[31] A. Riotto and M. Trodden, Ann. Rev. Nucl. Part. Sci. 49 (1999) 35; M. Dine and A. Kusenko, Rev. Mod. Phys. 76 (2004) 1; W. Buchmuller, R. D. Peccei and T. Yanagida, Ann. Rev. Nucl. Part. Sci. 55 (2005) 311.

[32] C. Grojean, G. Servant and J. D. Wells, Phys. Rev. D 71 (2005) 036001.

[33] S. Kanemura, Y. Okada and E. Senaha, Phys. Lett. B 606 (2005) 361. 